\title{
Making Sense of the World: Framing Models for Trustworthy Sensor-Driven Systems
}

\author{
Muffy Calder ${ }^{1, * \mathbb{D}}$, Simon Dobson ${ }^{2}$, Michael Fisher ${ }^{3}$ and Julie McCann ${ }^{4}$ \\ 1 School of Computing Science, University of Glasgow, Glasgow G12 8QQ, UK \\ 2 School of Computer Science, University of St Andrews, St Andrews KY16 9AX, UK; \\ simon.dobson@st-andrews.ac.uk \\ 3 Department of Computer Science, University of Liverpool, Liverpool L69 3BX, UK; MFisher@liverpool.ac.uk \\ 4 Department of Computing, Imperial College London, London SW7 2AZ, UK; jamm@doc.ic.ac.uk \\ * Correspondence: muffy.calder@glasgow.ac.uk
}

Received: 6 September 2018; Accepted: 12 November 2018; Published: 15 November 2018

\begin{abstract}
Sensor-driven systems provide data and information that facilitate real-time decision-making and autonomous actuation, as well as enable informed policy choices. However, can we be sure that these systems work as expected? Can we model them in a way that captures all the key issues? We define two concepts: frames of reference and frames of function that help us organise models of sensor-based systems and their purpose. Examples from a smart water distribution network illustrate how frames offer a lens through which to organise and balance multiple views of the system. Frames aid communication between modellers, analysts and stakeholders, and distinguish the purpose of each model, which contributes towards our trust that the system fulfils its purpose.
\end{abstract}

Keywords: models; assurance; sensor-driven systems; sensor networks

\section{Introduction}

Sensor-driven systems are everywhere: from transportation and buildings, to smart tags, power systems and environmental monitoring. They provide data and information that facilitate real-time decision-making and autonomous actuation, as well as enabling informed policy choices by service providers and regulators. The Internet of Things depends on robust, sensor-driven systems that can be trusted to deliver useful, correct, and timely information. Our vision is of smarter sensor-based systems of which stakeholders-from developers to scientists and policy makers-can ask deeper questions while being confident of obtaining reliable answers. However, can we be convinced that these systems do what we expect? Can their stakeholders ask deep questions and be confident of obtaining reliable answers? Are they trustworthy with respect to their requirements? This is especially difficult to determine, for example, when systems need to balance competing demands such as sampling rates required to answer a scientific question, timely actuation to ensure delivery of a service, and energy conservation when operating with constrained resources.

Given the ubiquity of sensor-driven networked systems, it is perhaps surprising that extracting reliable information from them remains far from straightforward: sensors consume power, they are noisy, they decalibrate, may become misplaced, moved, compromised, and generally degraded over time, both individually and as a collective. This is beyond traditional software engineering: uncertainty, failures, power and communication constraints pervade both the physical and digital environments in which these systems operate, and the sensors themselves, and is even crucial to some of the adaptive algorithms employed. Furthermore, sensors themselves are increasingly required to fulfil both housekeeping functions (reporting to the sensor provider) and multiple sensing functions (reporting to multiple applications). The sensor system may also be required to be smart, e.g., support, 
and exhibit increasing degrees of autonomy, self-awareness, and intelligence. These systems are core to many technologies intended to aid sustainability via smart cities, smart grids, smart farming etc., and yet their own reliability and sustainability is not well understood. Can we guarantee that the system will do what we expect? Can we, in good conscience, make use of the information being presented, and so trust the behaviour being exhibited? Without such assurances, no business or organisation will deploy complex, semi-autonomous, adaptive sensor-driven systems; no decision-maker will allow their decisions to rest on a possibly unstable foundation.

While we cannot engineer away issues such as uncertainty, failures, energy consumption etc., fortunately, sensor-driven system architecture tends to be specialised and constrained, usually, to an enhanced form of a sense and control loop. Thus, while assurance is still difficult, it is within the context of specialised concerns and architecture. It is our assertion that a crucial tool for addressing trustworthiness is the use of models, both at design time and run-time (e.g., online models): for specification; for explanation; and for exploration and prediction. Our assertion derives from our experience of research and development of a wide range of sensor-driven systems e.g., [1-9].

For any sensor-driven system, many models could be produced; we define two categorisations that are orthogonal yet overlapping, which help guide us. These two new concepts contribute to a principled modelling process by helping us to frame models of sensor-based systems and their purpose: frames of reference provide a way to organise and balance multiple perspectives and concerns, and frames of function identify component(s) within the system architecture that are the focus of attention. Together, they offer a lens through which to view and analyse the sensor-based system, which aids understanding of the purpose of that model and communication between modellers, analysts, and stakeholders. This allows us to distinguish and articulate the purpose of each model, contributing to our overall trust that the system fulfils its purpose and requirements. Figure 1 illustrates how different combinations of the frames can allow the multiple dimensions of focus to be reduced to make analysis more tractable, in terms of the development of models based on the questions we wish to ask of the system.

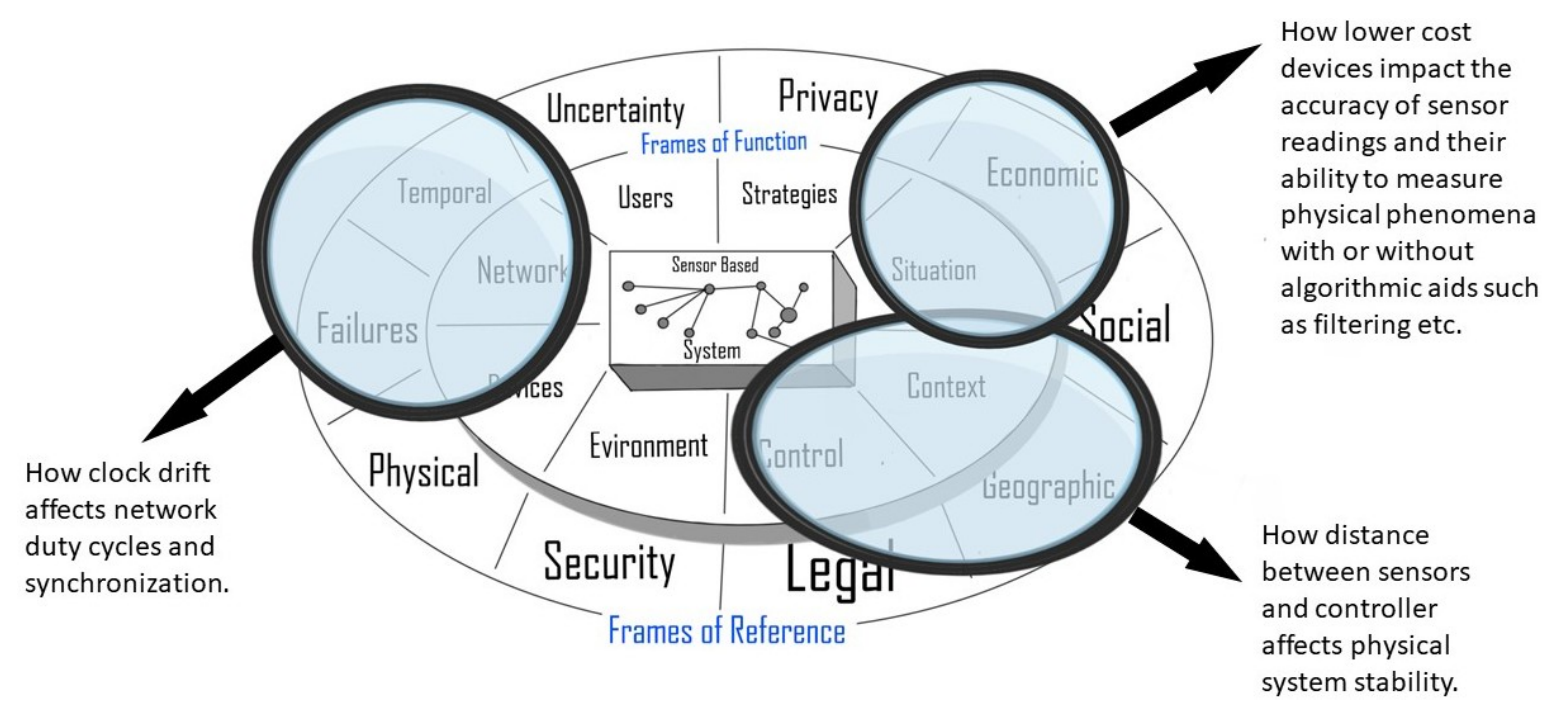

Figure 1. Frames of reference and function: a lens for modelling and analysis.

The following sections describe in more detail the two concepts that help us to frame model purpose, questions and analysis. In the next section, we review the motivation for models and in Sections 3 and 4 we define frames of reference and frames of function. In Section 5, we discuss how and when to use frames in modelling and in Section 6 we illustrate the new concepts by way of models for a smart water distribution network. We offer a short discussion in Section 7 and related work is discussed in Section 8; conclusions are provided in Section 9. It is important to note that this view 
provides an abstract methodology for design and development. While this must be subsequently populated with concrete languages and ontologies, these are typically very different for each scenario and so we will not expand on these here.

\section{Sensor-Based Systems and Their Models}

The concerns of uncertainty, failures, energy etc., that are inherent in practical sensor-based systems will always be present: these aspects cannot simply be engineered away and subsequently ignored. Further, the purpose, or mission, of the system may involve a number of trade-offs and compromises. A simple example involves controlling the duty cycle of a sensor node to preserve battery life. Efficient battery technology coupled with low-powered micro-controllers and transceiver devices have been key to the uptake of wireless sensors and now such systems can be placed in more remote and difficult to reach areas with minimal infrastructure required. Yet batteries, from a sensor systems point of view, have become an "Achilles heel" for the long-term sustainability and operation of wireless sensor systems. It is costly to replace batteries (and problematic if the sensors are inaccessible), they can be dangerous (lithium can cause fires), contribute to waste, and generally have a negative environmental effect. Further, energy harvesting is not always a solution either since energy storage is inherently inefficient and again storage cells last only for a limited number of years (think of mobile phone battery lifetimes). So, core to making sensor systems 'green' is energy management that typically implies at least duty-cycling and possibly adaptive duty-cycling. However, the latter might be constrained by a mission goal prescribing minimal sensing frequency-or might even be disallowed entirely because of the need for extraction of a regular time series. Without precisely-articulated requirements, it is impossible to know how this trade-off can be made in a way that does not compromise the efficacy of the sensor-based system. It is difficult to answer questions about energy source choice if we have not defined our goals and we do not have a way to explore design alternatives and their impact. Often such choices are implicit; formal models make them explicit, and thus amenable to analysis. Models are at the core of enabling stakeholders to have the confidence they need to trust in—and make decisions from—the information being returned from a sensor system.

Models are used extensively across science and engineering, providing insight into the behaviours of what already exists, predicting future behaviours of what might exist, and exploring the implications of the specifications and designs of what we want to exist and how and if they meet system requirements [10]. We do not repeat the arguments in support of modelling here, but note that recent research in pervasive and cyber-physical systems that tackles modelling, implementation, and deployment of artificial systems integrated within real-world environments, e.g., [11,12], is highly relevant. We draw on this work, for example from pervasive systems [13], where raw data from devices is processed as context, from which situations are inferred, and control and adaptation then depends on those situations, and we draw on cyber-physical systems to the extent to which they concentrate on the modelling and interaction of software with physical scenarios, typically using hybrid systems analysis and novel models of programming, as advocated in [12].

In [14], Lee refers to cyber-physical systems as the "fundamental intellectual problem of conjoining the engineering traditions of the cyber and the physical worlds"; he also sees models as being central to the study of such cyber-physical systems, noting that "software abstractions were not created for cyber-physical systems. They were created to run payrolls". We agree: we do not yet have the best abstractions and concepts for sensor-driven systems. Sensor systems dwell within other systems: water distribution systems, smart grid systems, industrial control systems, environmental systems and all of these envelop the sensor systems impacting on them and being impacted by them. One concrete example is of a sensor device that uses vibrations to carry out condition monitoring of an engine; it is responsible for interpreting the vibration and temperature signals and to quickly inform the actuator to slow the operation of the engine when either the temperature or vibrations reach certain threshold levels. This aims to maximise the lifetime of the engine, but revolution rates cannot fall below the 
engine efficiency requirements. The sensor device uses a piezoelectric harvester to obtain energy from the engine, yet when the vibrations lower the energy opportunity falls, and a dip in voltage can affect the sensor reading accuracy, cause clock drift, etc. The sensor node can provide guarantees that the energy levels are maintained, but is this at the cost of engine longevity? What we have described here consists of many sub-systems, the cyber computing context, the engine itself, its environment, the regulatory framework, etc. The nature and complexity of such hierarchical systems means that no single model can effectively cover everything. Therefore, we agree with other researchers investigating this subject that no one model fits all, however there is a lack of research in articulating how we bring these models together in a comprehensive yet tractable manner; this paper aims to address this omission.

The problem we address is how, from a statement of system requirements and stakeholder concerns, and an understanding of system architecture, we can derive both models and questions for analysis, as well as interpreting the answers, to assure stakeholders. Work in [10] reminds us of the importance of clarity about model purpose; and to be clear about what a model is and what it is not. To that end, frames improve the modelling and analysis process by enabling us to organise models and analysis and the ways they contribute to assuring system requirements and stakeholder concerns. Our goal is not a formally defined process, rather we offer these concepts as an aid to, or framework for, model development.

\section{Frames of Reference}

Stakeholders often have very different (possibly competing) perspectives on the key abstractions and assumptions about a sensor-based system. For example, developers may be focussed on the constraints (e.g., energy consumption) whereas users may be focussed on the services provided. Furthermore, the physical and digital environments in which sensor-based systems operate are inherently complex, and so it can be difficult to see how the different perspectives can be managed effectively. We define a frame of reference as a context in which measurements, judgements and interpretations can be made. Each frame articulates a different perspective and the dimensions and measurements specific to that perspective. Our definition aligns with standard dictionary definitions such as a set of criteria or stated values in relation to which measurements or judgements can be made; the overall context in which a problem or situation is placed, viewed, or interpreted; the observer interprets what he sees in terms of his own cultural frame of reference; we refine the concept further by enumerating the following frames relevant to sensor-driven systems.

- Geographic: spatial and topological relationships between sensors. Typically, these may be static networks (e.g., because the sensors are fixed to lampposts or in the ground), or dynamic networks (e.g., because they are fixed to mobile people, animals or objects), or on a fluid surface or within a flow in a pipeline. There may be many such relationships, one of which may be the data communication between sensors and controller(s), e.g., in star topology, peer to peer, etc.

- Physical: aspects of the environment in which sensors operate, be it physical or digital, which may affect system behaviours, e.g., physical aspects such as temperature, wind speed, may affect degradation of devices and other system components.

- Failures: relationships between the components that can fail, degrade, or operate incorrectly, including fail-safe mechanisms and redundancies.

- Economic: quantitative aspects of resources and their consumption, production and discovery. Example resources include energy, money, and digital data; typical measurements include maintenance costs, constraints on data buffers and communication bandwidth.

- Legal: deontic concepts such as obligations, permissions and responsibilities for different system components and human users.

- Security: vulnerabilities, threats and their mitigations, such as access controls preventing unauthorised entry to a system and encryption methods that encode data so it can only be 
accessed via keys. These vulnerabilities include those presented by multi-tenant architectures and the spatial factors of access, for example, close-proximity and remote-proximity.

- Privacy: anonymity, identity, authentication of personally identifiable information, and controls on intended and unintended disclosures.

- Social: communication and interaction between humans involved in the system, and between humans, the physical/natural world, and the underlying technologies. Usability and cognitive dissonance are key concepts. For example, GPS drift can cause cognitive dissonance and lack of trust in a system when users believe they are stationary yet their GPS coordinates are fluctuating.

- Uncertainty: what are the acceptable bounds of uncertainty for various aspects of the system, and how are the bounds qualified, quantified, and related to each other.

- Temporal: aspects of timing and computation paths that are relevant to system purpose, from simple temporal orderings to hard real-time constraints, as well as expected certainty of the model over time. For example, weather forecasting becomes less certain the further we look into the future, and navigation models become less precise as we move away from the position where we last verified a location.

We now turn our attention to the question of sensor-rich system architectures.

\section{Frames of Function}

Figure 2 illustrates a typical system software architecture in a smart, sensor-based system: the functional components and data flows. Components may relate directly to physical entities (e.g., devices) or be conceptual (e.g., situation, control). On the left, we have a standard sense and control loop, whereas on the right we have network communication, semantic and adaptive/autonomous capabilities, and above we have the physical/digital environment and human users. We define a frame of function as a component within this architecture, which we describe as follows (in simple systems, some of these components may not be present).

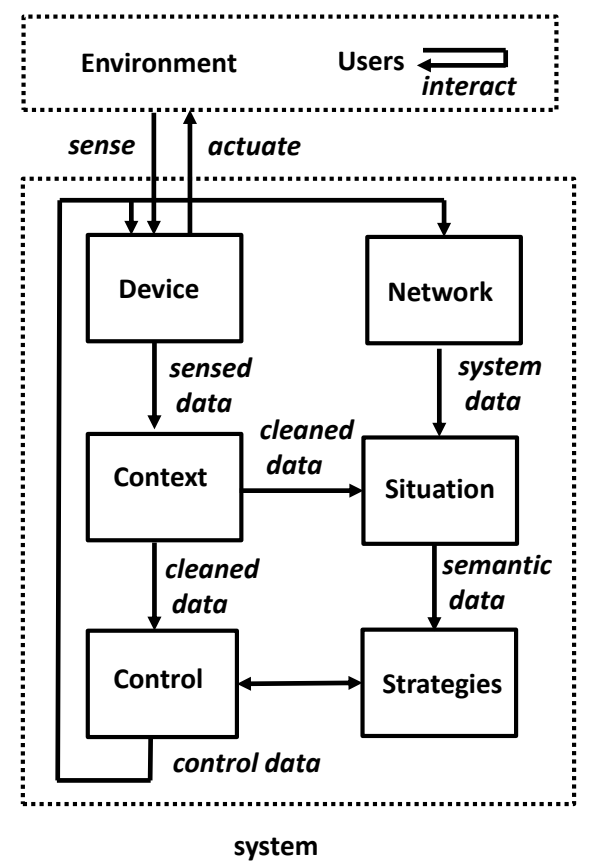

Figure 2. Typical sensor-driven system architecture: functional components and data flows.

- Devices interact directly with the environment, for sensing and actuation, they may also have a housekeeping role. Raw data from devices are processed as context, from which situations 
are inferred; control and strategies (and possibly adaptation) depend on those situations. There may be impacts of the physical environment on devices, e.g., degradation due to wear and tear, or effect of temperature on performance.

- Context refers to how raw signals from devices are processed into reusable computer data. This is typically a syntactic transformation that may, for example, include data smoothing, outlier rejection, and the extraction and maintenance of confidence intervals. We often refer to data that has been processed in this way as cleaned data.

- Control embodies the purpose of the system through control of individual devices and the network. For example, control may include powering down individual devices for periods of time, or changing communication rates.

- Network specifies how and when devices interact with each other, and with the application (through control). System data refers to operational data such as network status. Communications protocols are fundamental and usually depend on properties of the underlying sensor topology, e.g., star topology, peer to peer, and properties such as requirements for consistency between adjacent sensors; these properties may be dynamic. Sensor topologies may be modelled by metric or topological spaces. Models may also include representation of the impact of the physical environment on sets of devices and/or communications links.

- Situation refers to how data is interpreted semantically from context, typically by an inference process [15] that is implemented in software. For example, a data item might be classified as a known or a previously undetected event, or for a given time series, an inference process may infer semantic quantities such as "getting hotter" or "approaching a dangerous level".

- Strategies are how the software uses self-reflection (e.g., adaptation, autonomy) to achieve its mission and purpose, including node-level, network-level and application-level adaptations. Strategies may be tailored to the behavioural envelopes for individual nodes and ensembles. Behavioural envelopes can change, usually triggered by situation changes, and adaptation means that devices may be updated, enhanced or reconfigured. There is usually a close relationship between control and strategies, control typically depending on a mixture of strategies and (cleaned) data.

- Environment includes the laws and problem descriptions of aspects of the environment, be it physical or digital, which affect the function and purpose of the system. These come from natural science, engineering, regulations, and so on. For example, this might include analytical models such as differential equations, or statistical distributions, simulations, and specifications of safe and unsafe thresholds.

- Users: If human users are involved in the system and interacting with devices (e.g., wearing sensors), their behaviour can be considered as part of the environment. Alternatively, we may require more explicit consideration of relevant aspects of their behaviour. For example, this might include interactions between users and devices, or interactions between users, based, for example, on proxemics - theories of how people use interpersonal distance to understand and mediate interactions with others [16].

\section{How and When to Use Frames}

We propose a pragmatic, semi-structured process for employing frames, based on plain text. Clarity about what is in scope, as well as what is not, can help us to focus on the purpose of each model and analysis, and identify any gaps we have overlooked. We have deliberately chosen not to define a formal meta-level framework for modelling: our experience tells us that what is required most urgently is clarity about the purpose and dimensions of a model, rather than another formal framework that is only used by a small community of experts. We make no assumption about the type of model, e.g., continuous, discrete, stochastic, state-based, event-based, logic-based, agent-based etc., but do assume there are established techniques and tools for formal analysis. The following is a guide to using frames. 
Frames are used when

- $\quad$ selecting the modelling formalism and developing a model,

- selecting questions that can be asked of the model and encoding those questions using the analysis techniques for that formalism,

- interpreting and communicating answers from model analysis to stakeholders,

- documenting a model for possible future re-use and/or certification,

- reviewing model coverage: identifying and explaining gaps in a set of models, for example, which frames of reference/function are missing and why.

The selection and combination of frames (reference and function) for each model involves discussion between modellers, analysts, and systems developers. While the primary focus may be a particular set of frames, a model may need to have abstract representations or requirements derived from one set of frames, in order to represent and analyse in more detail another set. For example, we may require representations about the uncertainty of devices and their control, in order to model uncertainty in the network communications.

\section{Modelling with Frames: Examples from a Smart Water Distribution Network}

The following example is taken from our experience of working with smart water distribution networks that monitor and control pumping, valves, and communication. In these systems, treated water must be kept moving at sufficient hydraulic pressure to maintain the treatment, yet should not put undue pressure on the pipes. Pumping treated water reservoirs to supply zones and storage tanks consumes most of the energy budget for a utility. Smart use of tanks and reservoirs, and shifting pumping schedules to cheap tariff periods, can result in savings and so impact how water systems remain sustainable.

The purpose of a smart water distribution network is to minimise pumping costs, minimise pipe degradation and leakage, and satisfy customer demands over water pressure and quality. The systems includes water reservoir(s), water tanks, flow meters, pressure sensors, motorised valves, and pump(s). Data communication-between nodes (sensors) and pump, and from pump to valve-may be subject to transmission delays and bandwidth constraints.

Typical stakeholder questions include the following: What is lowest pressure that can meet demand and keep water clean? What is the highest pressure that minimises pipe damage? What is the minimal data rate that meets the legal requirements for reporting leaks? Under the assumption of no failures, does the proposed pumping schedule meet customer demand over a $48 \mathrm{~h}$ period? How can resource usage be reduced, e.g., energy, in delivering water sustainably, given changing environmental conditions?

Modelling this type of system is challenging because we have continuous behaviour of fluid flows and motorised valve (devices), stochastic behaviour of a digital communication (network) and pipe degradation, and discrete behaviour of the control software. There are periodic and aperiodic events. Note that there are no human users nor personal (e.g., billing) data in the system.

We describe informally below four models that are under development based on Waterbox-an experimental platform [1] developed at Imperial College. We list the primary frames, the modelling and analysis techniques. Details of these models will be reported in further technical papers.

\subsection{Dynamics of Fluids and Tolerances}

Frames of reference: Physical, Geographic, Temporal Frames of function: Control, Environment

This model represents the dynamics of fluids and physical control with differential equations based on control theory; embedded in them is the relevant physics of the physical water system (hydraulics etc.). In the cyber-physical systems (CPS) community, this would be described as 
representing the physical model. The purpose of this continuous model is to investigate under what circumstances the system control ensures that the overall system meets the requirements of customer demand and there are no overflows. Delays in control signals can be as detrimental to the system converging as not being able to detect the state of the water system. The impact of delays (from communications or other sources) has to analysed, for each particular controller design. Specifically, the purpose of each of these models (for each controller design) is to determine control tolerances: What are the maximum message delays and message loss that can occur in the system while still fulfilling the requirements of customer demand with no overflows? For how long is this maintained? What is the degree of disturbance that can be tolerated? Within these frames, we are not interested in the causes of delays: it is not important whether this is caused by node failure, bandwidth issues or packet loss. However, the number of devices reporting to a centralised controller can delay its calculations; therefore, the processing capacity of the controller is very relevant. Considering the Temporal frame of reference, there would not be many changes in the models over time-except where the water network itself is expanded or trends in demand change due to, for example, a new hospital being built that would cause an impactive change in demand calculations and therefore control schemes. Analysis of the equations is by simulations, and typically uses standard control system development tools such as Simulink [17].

\subsection{Impact of Data Communications}

Frames of reference: Economic, Uncertainty, Failures

Frames of function: Network, Devices

Here, the resource in the Economic frame is data; the stochastic behaviours in the Uncertainty frame arise from packet loss and delay. The purpose of this model is to investigate different protocols and data routing algorithms for data communication in the network, the different ways to deal with the bandwidth and device data buffer constraints, and when/how individual devices contribute to delays of packets. We assume that we have already determined the tolerances of the control function, namely maximum message delays and message loss that the control function can tolerate and still fulfil requirements of customer demand with no overflows etc., in the model above (Section 6.1). Most control systems utilise a TDMA style MAC protocol (as opposed to CSMA and multi-hop approaches), because of the tight timing orchestrations that ensure when a node sends is more deterministic and therefore packet reception times are more predictable. We examine the use of LoRa, a standard low-powered wide-area communications to relay data to the base-station; LoRaWan (its MAC) can provide guarantees of sensor data deliveries due to its scheduled TDMA nature. We can describe this as the "cyber" side of the CPS equation, and we can model this with a probabilistic discrete space model. We use probabilistic temporal logic properties to express whether the tolerances of message delays and loss will always (or sometimes, and under which circumstances) be met, and use the model-checker PRISM [18], to prove them, or if not, to indicate the circumstances under which they fail to be met.

\subsection{Energy Consumption}

\section{Frames of reference: Economic, Geographic}

Frames of function: Network and Strategies

Here, the resource in the Economic frame is energy and the aim is to show (or not) that the battery operational lifetime lasts two years or more (to minimise the maintenance costs of the sensor network), and at the same time the quality of the data is maintained such that there is at least one data reading available every $15 \mathrm{~min}$. We assume that the network uses low-powered wide-area communications to relay data in a star topology to a single base-station (Geographic frame of reference). Compressive sensing techniques are used to adapt the sensing rate down when data values indicate that the next sensor reading is not necessary (this technique can interpret data trends and predict that, for example, 
temperature will not change more than a degree in the next time slice), which is examined under the Strategies frame of function. The model is again probabilistic discrete state, but in this case the focus is not on the probabilities per se but on the rewards (i.e., costs) that are associated with both states and transitions. Rewards are a feature of the PRISM [18] model checker, which is used to examine reward-based temporal properties that express assurances about battery lifetime, while meeting data sampling requirements. Note that to offer stronger assurance, we may in future add the Situation frame of reference and make inferences about energy consumption over sets of observed traces over time, and use these to fine-tune the rewards.

\subsection{Detecting Security Attacks}

Frames of reference: Security Frames of function: Context, Situation

Finally, we consider how two types of Security attack on the communications network impact the control operation. Jammed communication acts as a delay and spoofing can inject false data. In both cases, the result is incorrect system (state) and semantic data that causes the system to function incorrectly. For example, these attacks can cause pipe bursts due to constant actuation causing water pressure hammers, or can allow water to overflow reservoirs. We examine (cleaned) sensed data and system in the Context and Situation frames, using machine learning, to detect "abnormal" sensing and networks communications. We can infer stochastic models of clusters of "normal" behaviour and variances from sampled event streams, consisting of both periodic data sensing events and aperiodic control events. We then use the models at runtime, as online monitors to detect "abnormal" sensing communication, by comparing the observed data streams from the system with the expected streams.

\subsection{Model Coverage}

The simple tables in Figure 3a,b summarise the frames employed in the four models above: 1 refers to model in Section 6.1, etc. Frames label the rows: frames of reference are on the left, frames of function are on the right. An entry indicates that the frame was used in that model, e.g., Geographic frame of reference was employed in models 1 and 3, the Environment frame of function was employed in model 1. The Social and Privacy frames of reference are omitted as they are not relevant to this system. We do not expect a uniform distribution of frames and it is not surprising that the Geographic and Economic frames of reference and Network frame of function are addressed in more than one model—these are key frames for this system. We can see quickly that we have not addressed the Legal frame of reference. This is deliberate, for this system, because burst detection is straightforward (e.g., from flows) and sub-second notification is not required.

\begin{tabular}{r|c|} 
Frame of reference & model \\
\hline Geographic & 1,3 \\
Physical & 1 \\
Economic & 2,3 \\
Security & 4 \\
Uncertainty & 2 \\
Temporal & 1 \\
Failures & 2 \\
Legal & -
\end{tabular}

(a) Frames of reference.

\begin{tabular}{r|c|} 
Frame of function & model \\
\hline Environment & 1 \\
Device & 2 \\
Context & 4 \\
Control & 1 \\
Network & 2,3 \\
Situation & 4 \\
Strategies & 3
\end{tabular}

(b) Frames of function.

Figure 3. Frames of reference (a) and function (b) employed in models 1-4.

\section{Discussion}

The variety of models required for this one system demonstrates the breadth and complexity of sensor-driven networked systems and their requirements. In this case, no single model would be 
tractable, or comprehensible: each model has a purpose and analysis of that model contributes to our overall understanding and trust that the system fulfils its requirements. Frames help us to distinguish and articulate the purpose of each model, and also to articulate the absence of a model.

We recognise that models are just a single, albeit important, component of assurance of sensor-driven systems: modelling and analysis needs to be coupled with software development, testing and analysis of actual deployments. While techniques for context and situation recognition and inference have, and are, being researched extensively, especially in the context of "big data", we suggest that there are several new challenges specific to sensor-based system modelling. For example:

- How can historical data be used to calibrate models that have not been inferred from data, for the analysis of system play-back, and also to aid predictions of future system behaviour?

- How can we use model predictions as inputs to control, and use sensed contexts and situations to calibrate model predictions? For example, can we use model predictions to modify system goals, to drive sensor change, and detect data anomalies?

- Over time, context and situations that have been observed and inferred may be compared with those predicted by standard models based on laws of physics, e.g., observed (sensed) water levels are compared with predicted water levels. However, we may find that the observed levels are not as predicted. How should we modify the (possibly long established) physics models when they do not align with actual sensed data?

- What can we learn from sensor-driven systems that fail, as well as succeed? Too often, only successful applications are recorded. What is the role of modelling in failed systems? A good example of a failed application is the remarkably honest assessment of a 150 wireless sensor node application for precision agriculture reported in [19]. A culture of reporting failures, and analyses thereof (e.g., the recent FAILSAFE workshop [20]) will only strengthen the role for improved modelling and also for sensor-driven systems.

\section{Related Work}

The problem of identifying and dealing with multiple perspectives, or views, of a system, particularly during software development, is well known in software engineering. We attempt here to situate our work in that context, noting that we do not give a comprehensive literature review, but rather select key related work.

Nearly thirty years ago, Somerville introduced viewpoint analysis [21], where a viewpoint is defined informally as a "way of looking at the system". Viewpoints are very wide ranging: there may be tens or hundreds of viewpoints for a system, the exact number and nature depends on the system. There is some categorisation, e.g., there are functional viewpoints and non-functional viewpoints. Very loosely, we can say that our frames of function correspond to the former and our frames of reference to the latter. Further comparison is difficult because Sommerville's viewpoints cover all the breadth (and depth) of software systems; we are concerned with a specific domain (sensor-driven systems), and so we can be much more prescriptive about our frames.

Subsequent research on views has been more rigorous, such as contract-based design and multiple viewpoints [22-24], which aim to support distributed design of different aspects of the system; the key results are generic mathematical models that formalise contract theories and meta-theories. We have deliberately chosen not to formalise processes, e.g., how to integrate models, or introduce new modelling techniques. Rather, our aim has been a pragmatic, semi-structured process to improve model development and interpretation and communication of results.

Several researchers have recognised the difficult problem of framing, for example, problem frames [25] and experimental frames [26,27]. The former presents a set of concepts to be used when gathering requirements and creating specifications for software; these include specific frames of required behaviour, commanded behaviour, information display, etc. Again, while these are useful in the context of general software development, they are focussed on requirements and design, and 
do not meet our specific needs of articulating model purpose for sensor-driven systems that operate in a cyber-physical world. The latter is defined specifically for discrete event simulation models and addresses the problem of characterising a set(s) of circumstances under which a model is to be observed or experimented with. This includes specifying the mean rate of arrivals, seeds for the random number generators etc. This provides a useful, but narrow lens with which to view models, and apart from assuming the model is event based, it disregards any domain-specific aspects such as what the events represent. We expect experimental circumstances to be part of the dimensions of a frame, or pair of (reference and function) frames. For example, rates of communication bandwidth would be specified within the economic and network frames, rates of sensor failures within the failure and device frames, and message loss rates within the failures and network frames. Finally, there are few explicit concepts for modelling sensor-driven systems, apart from those we mentioned earlier for pervasive computing and cyber-security, though we note that [11] classifies properties as local spatial (entities are in pre-defined structural patterns), global spatial (entities are arbitrarily distributed in space), and temporal, which loosely correspond to our geographic and temporal frames of reference.

Can our concepts add new insights into existing models of sensor-based systems? For example, the Ptides and Ptolemy models described in $[12,14]$ refer to interplays between physical models (i.e., Environment), sensors, actuators (i.e., Devices), computation (i.e., Control), and Network communication. The Failures and Temporal frames of reference are predominant, with an emphasis on timing and scheduling. Additionally, in [12] a modal, hybrid model in Ptolemy II is proposed for improved adaptation; we would classify this as focussing on the Strategies frame. Future work could determine how our frames help with modifications to these systems and/or how the models could be extended to incorporate assurance over other frames of reference.

\section{Conclusions}

Sensor-driven systems will become increasingly significant over the coming decades, supporting evidence-based decision-making in the face of global challenges such as environmental change, food production, internet of things and autonomous vehicles. However, progress will be undermined by our inability to assure both decision-makers and users of the integrity and timeliness of the information being provided and the decisions taken. Without such assurance, increasingly smart infrastructures will become unpredictable and unacceptable, and the ability of state, scientific, and industrial actors to leverage the benefits of sensing and autonomy will be severely restricted.

Modelling is a crucial part of establishing trust, and models are applicable not only at design time, but also during deployment, as a system is running. We have introduced two new concepts: frames of reference and frames of function, which provide an abstract methodology for design and and development. They improve the modelling and analysis process by helping us to distinguish and articulate the purpose of each model, which in turn helps us to deal with, communicate, and assure stakeholders about the competing demands of understanding the sensed world, the computation, communication, and self-reflection within a sensor-based system, and the ways in which the devices interact with the sensed world.

We have outlined how these concepts are being employed in an example system that we are working on; demonstration of the full benefits of these models is the focus of further technical papers. Future work will include further demonstration and development of frames of reference and function, including the concrete languages and ontologies needed, through case studies. The case studies will be based on increasingly complex end-user applications that cover a wide range of frames and include sensor-driven systems that fail, as well as succeed.

Author Contributions: All authors contributed to the ideas contained in this paper and the manuscript was written and edited by all authors.

Funding: This work is supported by the Science of Sensor System Software programme grant, funded by the EPSRC (Engineering and Physical Sciences Research Council), EP/N007565/1.

Conflicts of Interest: The authors declare no conflict of interest. 


\section{References}

1. Kartakis, S.; Abraham, E.; McCann, J.A. WaterBox: A Testbed for Monitoring and Controlling Smart Water Networks. In Proceedings of the 1st ACM International Workshop on Cyber-Physical Systems for Smart Water Networks, Seattle, WA, USA, 13-16 April 2015; pp. 8:1-8:6.

2. Kamal, A.R.M.; Bleakley, C.J.; Dobson, S. Failure Detection in Wireless Sensor Networks: A Sequence-based Dynamic Approach. ACM Trans. Sens. Netw. 2014, 10, 35:1-35:29. [CrossRef]

3. Fang, L.; Dobson, S. In-network Sensor Data Modelling Methods for Fault Detection. In Evolving Ambient Intelligence; Springer: Cham, Switzerland, 2013; pp. 176-189.

4. Fisher, M.; Dennis, L.A.; Webster, M.P. Verifying Autonomous Systems. Comm. ACM 2013, 56, 84-93. [CrossRef]

5. Konur, S.; Fisher, M.; Dobson, S.; Knox, S. Formal verification of a pervasive messaging system. Form. Asp. Comput. 2014, 26, 677-694. [CrossRef]

6. Calder, M.; Gray, P.; Unsworth, C. Is my configuration any good: checking usability in a sensor-based activity monitor. Innovat. Syst. Softw. Eng. 2015, 11, 131-142. [CrossRef]

7. Benford, S.; Calder, M.; Rodden, T.; Sevegnani, M. On lions, impala, and bigraphs: modelling interaction in physical/virtual spaces. ACM Trans. Human-Comput. Interact. (TOCHI) 2016, 23, 9:1-9:56. [CrossRef]

8. Ye, J.; Stevenson, G.; Dobson, S. Detecting abnormal events on binary sensors in smart home environments. Pervasive Mob. Comput. 2016, 33, 32-49. [CrossRef]

9. Jackson, G.; Kartakis, S.; McCann, J.A. Accurate Models of Energy Harvesting for Smart Environments. In Proceedings of the 2017 IEEE International Conference on Smart Computing, SMARTCOMP, Hong Kong, China, 29-31 May 2017; pp. 1-6.

10. Calder, M.; Craig, C.; Culley, D.; de Cani, R.; Donnelly, C.A.; Douglas, R.; Edmonds, B.; Gascoigne, J.; Gilbert, N.; Hargrove, C.; et al. Computational modelling for decision-making: where, why, what, who, and how. R. Soc. Open Sci. 2018, 5, 172096. [CrossRef] [PubMed]

11. Tsigkanos, C.; Kehrer, T.; Ghezzi, C. Modeling and verification of evolving cyber-physical spaces. In Proceedings of the 11th Joint Meeting on Foundations of Software Engineering, ESEC/FSE, Paderborn, Germany, 4-8 September 2017; pp. 38-48.

12. Derler, P.; Lee, E.A.; Sangiovanni-Vincentelli, A.L. Modeling Cyber-Physical Systems. Proc. IEEE 2012, 100, 13-28. [CrossRef]

13. Coutaz, J.; Crowley, J.; Dobson, S.; Garlan, D. Context is key. Comm. ACM 2005, 48, 49-53. [CrossRef]

14. Lee, E.A. The Past, Present and Future of Cyber-Physical Systems: A Focus on Models. Sensors 2015, 15, 4837-4869. [CrossRef] [PubMed]

15. Ye, J.; Dobson, S.; McKeever, S. Situation identification techniques in pervasive computing: A review. Pervasive Mob. Comput. 2012, 8, 36-66. [CrossRef]

16. Greenberg, S.; Marquardt, N.; Ballendat, T.; Diaz-Marino, R.; Wang, M. Proxemic Interactions: The New Ubicomp? Interactions 2011, 18, 42-50. [CrossRef]

17. Simulink. Available online: https://uk.mathworks.com/help/simulink (accessed on 12 November 2018).

18. Kwiatkowska, M.; Norman, G.; Parker, D. PRISM 4.0: Verification of Probabilistic Real-time Systems. In Proceedings of the 23rd International Conference on Computer Aided Verification (CAV'11), LNCS; Springer: Berlin/Heidelberg, Germany, 2012; Volume 6806; pp. 585-591.

19. Langendoen, K.; Baggio, A.; Visser, O. Murphy Loves Potatoes: Experiences from a Pilot Sensor Network Deployment in Precision Agriculture. In Proceedings of the 20th International Parallel and Distributed Processing Symposium (IPDPS), Rhodes Island, Greece, 25-29 April 2006.

20. First ACM International Workshop on the Engineering of Reliable, Robust, and Secure Embedded Wireless Sensing Systems (FAILSAFE). Available online: http:/ / wp.doc.ic.ac.uk/failsafe (accessed on 12 November 2018).

21. Sommerville, I. Software Engineering, 4th ed.; Addison Wesley: Boston, MA, USA, 1992.

22. Von Hanxleden, R.; Lee, E.A.; Motika, C.; Fuhrmann, H. Multi-View Modeling and Pragmatics in 2020: Position Paper on Designing Complex Cyber-Physical Systems, LNCS; Springer: Berlin/Heidelberg, Germany, 2012; Volume 7539.

23. Hennicker, R.; Ludwig, M. View-Based Development of a Simulation Framework for Multi-disciplinary Environmental Modelling, LNCS; Springer: Berlin/Heidelberg, Germany, 2012; Volume 7539. 
24. Benveniste, A.; Caillaud, B.; Passerone, R. A Generic Model of Contracts for Embedded Systems. arXiv 2007, arXiv:0706.1456.

25. Jackson, M. Problem Frames: Analysing and Structuring Software Development Problems; Addison-Wesley: Reading, MA, USA, 2001.

26. Zeigler, B. Theory of Modelling and Simulation; Wiley: New York, NY, USA, 1976.

27. Daum, T.; Sargent, R.G. Experimental Frames in a Modern Modeling and Simulation System. IIE Trans. 2001, 33, 181-192. [CrossRef]

(C) 2018 by the authors. Licensee MDPI, Basel, Switzerland. This article is an open access article distributed under the terms and conditions of the Creative Commons Attribution (CC BY) license (http://creativecommons.org/licenses/by/4.0/). 